\title{
Surveillance Resilience Design Designing for Protecting the Personal Identity in the Age of Surveillance
}

Massimo Bianchini

Politecnico di Milano

massimo.bianchini@polimi.it

ORCID 0000-0003-1438-4976
Rei Morozumi

Independent Researcher

reimorozumi@gmail.com

\begin{abstract}
Ubiquitous Surveillance refers to the unilateral collection of data on people through sensors (Oulasvirta et al., 2012). It is a phenomenon under-explored and under-practised within the design discipline. The proposed work ${ }^{1}$ explores the resilience to surveillance by framing it from the design perspective. It starts with a literature review to outline the Age of Surveillance and analyses approaches and tools to address such complex and controversial topics, focusing on design for Surveillance Capitalism (Zuboff, 2018). Finally, the article outlines the principles and values of Surveillance Resilience Design.
\end{abstract}

\section{Keywords}

Ubiquitous Surveillance Surveillance Capitalism Datafi d citizens Artificial I telligence Internet of Things (IoT) 
The principle of Privacy By Design (PBD), recently included in the General Data Protection Regulation, or GDPR (2016), introduces a human-centred design approach in the field of personal data protection. This principle requires organizations to develop designs that include the right tools and methods to protect personal data. While legislators and scholars have widely investigated the concept of privacy, the idea of design (within the PBD context) has received much less attention. There is essentially a lack of a broader view of the contribution of design to the development of active approaches and tools to solve privacy problems (Wong \& Mulligan, 2019). If the role of design in privacy has so far been little considered or explored, even less so is the related emerging issue of Ubiquitous Surveillance. Why? There may be several reasons for this. Among them is the fact that this is a strongly technology-based field, in which dominant engineering approaches assume that privacy is predefined and not something that requires to be challenged at the design level, either in predicting problems or in setting up technological systems. The other fact is that design expansion into a relevant field such as Human-Computer Interaction $(\mathrm{HCl})$ is relatively recent (Wong \& Mulligan, 2019). What is certain is that only in recent times design - especially critical design and partially service and User Experience (UX) design - started to explore this topic. And it has done so more in a dimension of social-political activism and criticism of Surveillance Capitalism (Fuad-Luke, 2009) than in the design of producible solutions.

The article stimulates a scientific and cultural debate on the relationship between design and Ubiquitous Surveillance, starting from two research questions: can design play a role within the surveillance society? Can design stimulate innovative solutions for protecting personal identity?

\section{Exploring the Surveillance Capitalism to Design a Framework for the Surveillance Resilience}

Surveillance is the general ability to know individuals in-depth, understand personality, recognize emotions, and penetrate practically even in the unconscious part of the person. The fluidity of technologies that characterize loT allows the actors operating in the surveillance field to apply the rules used on the web in the real world, tracking and extracting behavioural surplus (Zuboff, 2018, p. 221). In addition to the development and installation of sensors in cities or environments, the proliferation of smart devices and smartphones themselves allows Artificial Intelligence (Al) to use these tools as sensors (Zuboff, 2018) capable of tracking and extracting data ranging from environmental data to data related to the user's behaviours, habits, and emotions even in real life. Moreover, computers can recognize a user's emotions in real-time through biometric recognition such as facial, voice, or heartbeat recognition (Khatchadourian, 2015). Thus, emotional and ubiquitous data are crossed, forming dynamic profiles (Schwartz, 2016). This aspect enables increasingly massive access to the unconscious and can
The article systematizes and reworks a literature review on the theme of Ubiquitous Surveillance initially developed within a thesis of the Master's Degree in Integrated Product Design at the School of Design of Politecnico di Milano, entitled SU.RE. TOOLS, Designing Surveillance Resilience (author: Rei Morozumi, supervisor: Massimo Bianchini, co-supervisor: Francesco Zurlo). 
predict behaviour solely through facial reactions. The new frontier of surveillance triangulates all data to increase accuracy and achieve omniscience over individuals, collecting a behavioural surplus to identify the intentions, moods, emotions, and people's inclinations. Various research has led to the analysis of so-called metadata, rather than lists about the preferences shared by users, arriving at the possibility of predicting traits of a user's personality with an error of only a tenth (Ferwerda et al., 2016).

The global growth of ubiquitous computation enables Ubiquitous Surveillance, an apparatus more relevant than ever in our society. Ubiquitous Surveillance is highly asymmetric. It knows every individual ubiquitously and profoundly while, on the contrary, individuals are often unaware that they are being monitored. Social sorting is a relevant consequence of Ubiquitous Surveillance (Bauman \& Lyon, 2013, p. 17). It is a type of discrimination that, based on profiling and behavioural predictions, discriminates people not only by race, gender, etc. but also according to lifestyle, bank account, tastes, personality, a phenomenon that has been defined as "rational discrimination" by O.H. Gandy (2016). Didier Bigo calls "Ban-opticon" the system that aims at normalization and uses these mechanics to separate what is defined as usual from what is not and is therefore excluded (Bauman \& Lyon, 2013, p. 58-60).

Behavioural data can be defined as "surveillance assets" or the raw material representing oil of surveillance capital in what Shoshana Zuboff calls Surveillance Economy (Zuboff, 2018). Within Surveillance Economy, users become a source from which info (the new raw material) is extracted to create behavioural predictions (the new products) through Als (the new means of production). Thus, Surveillance Capitalism is a market for future behaviour in which surveillance is itself a service. Nowadays, companies offer services that monitor, track, surveil, and predict on behalf of third-party companies to which they provide, for example, info about the financial reliability of the customers (Zuboff, 2018, p. 185).

In the emerging paradigm of the Surveillance Society, it becomes relevant to consider the transformation of citizenship patterns and lifestyles of individuals, re-discussing principles and values such as those attributed to privacy that in the Western culture is strongly related to the concept of autonomy (Henkin, 1974). DeCew (1997) describes privacy as a shield that protects us, whose value lies in the freedom and independence it guarantees us. A relevant aspect of the Surveillance Society concerns the role of algorithms and data. Using the term "dataism", Harari (2015) defines a phenomenon in which personal information is replaced by objective data, based on data and algorithms, and proposing in them and algorithms salvation. Therefore, they have more competence in deciding on personal life, always knowing what is best for the individual and the system. One of the natural consequences of a society that entrusts decisions to algorithms is the disappearance of chance, of surprise, as everything has been pre-studied and results from predictions and behavioural inductions (Kelly, 2016). Bauman defines this phenomenon as "adiaphorization". Adiaphorization is the separation between the system and personal morality caused by the automation technologies that effectively separate the person from the responsibility for his action. In a context where 
digitization has pushed into the real world, authentic identity and virtual identity are increasingly coinciding (Beck, 2016, p. 153).

Finally, it is crucial to understand the relationship between Surveillance Capitalism and post-humanism (Pötzsch, 2014; Kumar, 2019). The implications of Surveillance Capitalism and Ubiquitous Surveillance go beyond privacy and personal identity questions. In this perspective, we can talk about more advanced (and invasive) forms of security and care, about the deconstruction of panopticons (Mann et al., 2003; Galič et al., 2017) and the parallel theorization of alternative models of surveillance such as the "Oligopticon" (Latour, 2005), a partial but more efficient surveillance model.

The conceptual structure of a Surveillance Society can be represented as a "T" model Fig. 1. Emotional and ubiquitous data are crossed, forming dynamic profiles. The diagram, therefore, helps to identify one or more areas of interest and possible design opportunities.

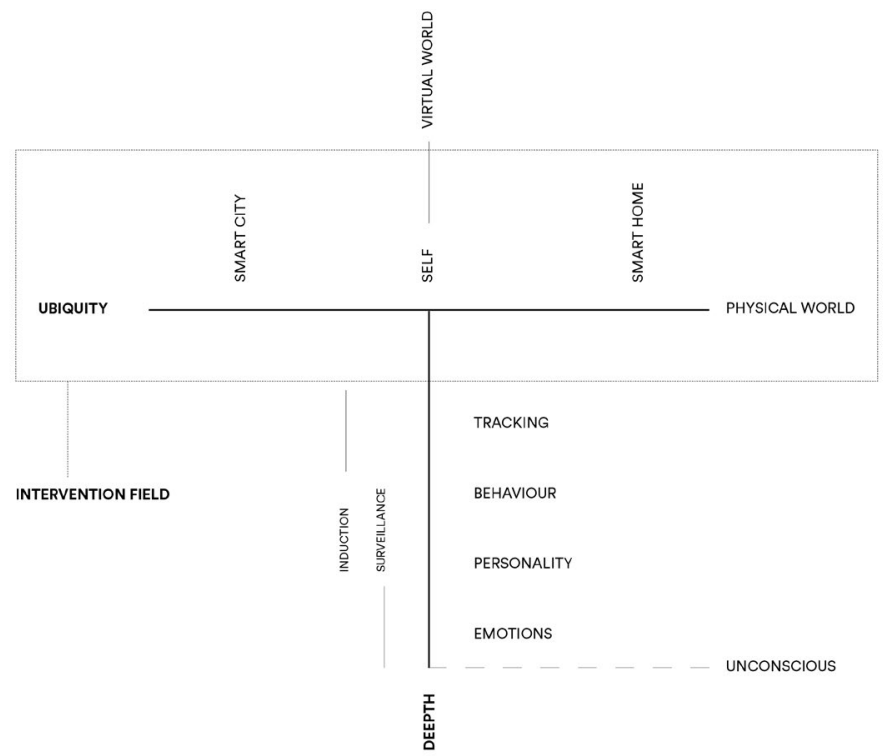

The issues identified during the exploratory phase, a series of relevant cases relating to projects dedicated to surveillance protection, were collected through desk research. Therefore, this research ranges from different creative disciplines and is characterized by different design and innovative approaches: innovative applications of an experimental nature or designed to raise awareness, results of scientific research, and marketed products. Forty-eight cases ${ }^{2}$ have been collected and systematized within a matrix Fig. 2 to visualize the various surveillance resilience projects.
The cases were identifi d and then mapped through desk research and sys-

tematized using summary sheets. The main criteria used were the presence of a project output (a prototype, a product-service, an installation, etc.), the participation of the case in relevant event, the publication of the case in technical or scientific journals or ther well-known information channels.

Fig. 1

The conceptual structure of the Surveillance Society. 


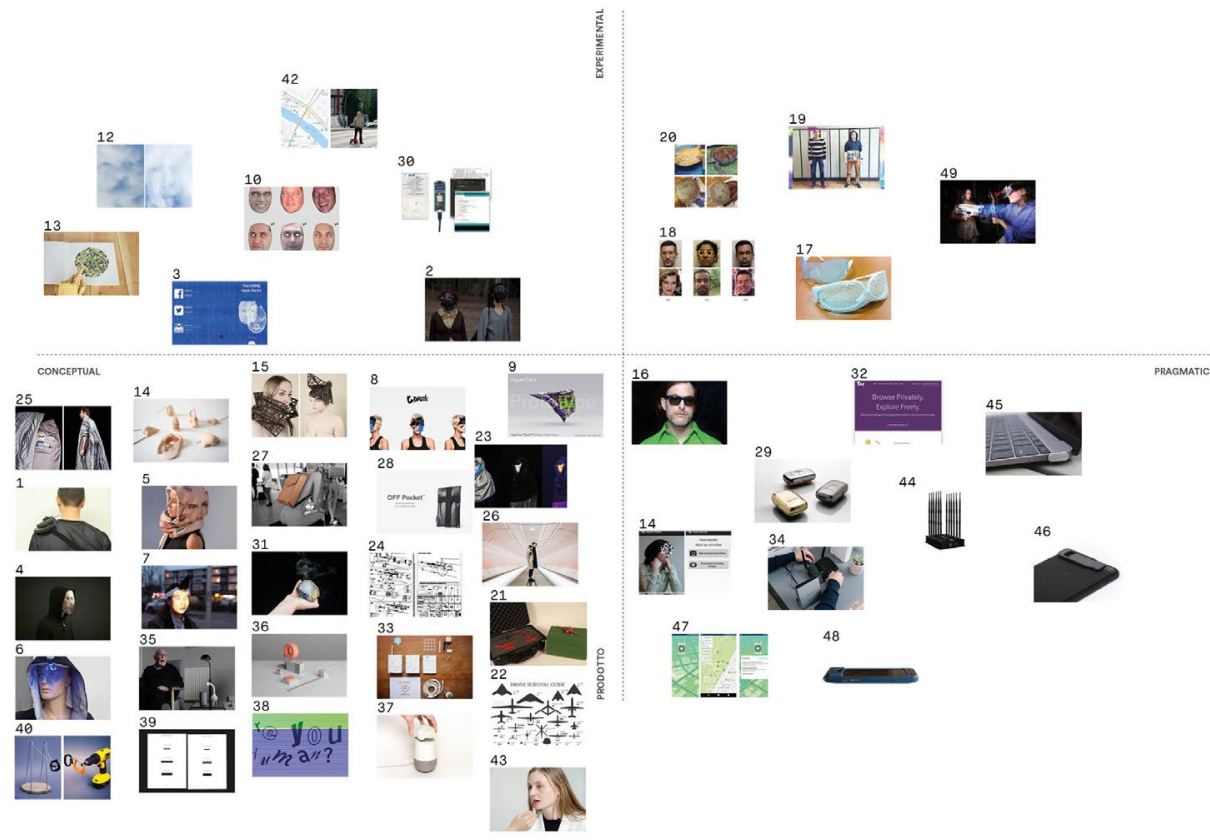

Fig. 2

The matrix with the surveillance approaches and related cases.

List of the mapped cases: 1 Surveillance Spaulder by James Bridle (2013)

2 Facial Weaponization Suite by Zach Blass (2011-2014)

3 The URME Hack Me Kit by Leo Selvaggio (2015)

4 Privacy Mask, Anonymous by Jip van Leeuwenstein - HKU (2017)

5 Anonymity Scarf by Sanne Weeker - HKU (2017)

6 Dazzle Coat by Joppe Basseling - HKU (2017)

7 Face Protector by Jing Cai Liu (2017)

8 CV Dazzle by Adam Harvey (2010)

9 HyperFace by Adam Harvey - HyperLabs

(2017)

10 You Can Encrypt Your Face by Sam Levigne (2017)
11 Cloud Face by Shinseungback Kimyonghun (2012)

12 FADTCHA by Shinseungback Kimyonghun (2013)

13 Tricking Biometrics by Alix Gallet (2016);

14 Face Dazzle by Petr Prokop (2015)

15 Anti-Nis Accessories by Lisa Cori and Catlin Morris - Fabrica (2013) 16 Refl ctcles by Scott Urban (2017)

17 Privacy Visors by NII Japan (2015)

18 Anti-Facial Recognition Eyeglasses by Carnegie Mellon University (2016)

19 Fooling Surveillance Cameras by KU Leuven (2019)

20 Fooling Neural

Networks in the Physica World by Lab Six (2017) 21 UAV Identific tion Kit 001 by James Bridle (2012)

22 Drone Survival Guide by Ruben Patern (2014) 23 Stealth Wear by Adam Harvey (2012)
24 Anti-Drone Canon by Marcel Coufreur Hku (2017)

25 CHBL - Jammer Coat by COOP HIMMELB(L)AU (2014)

26 Project KOVR by

Marcha Shagen and Leon Baauw (2016)

27 Faraday Backpack by Joppe Besseling HK (2017)

28 OFF Pocket by Adam Harvey (2011-2013)

29 Embracing Paranoia

Collection by Koncern

(2015)

30 Sky Lift by Adam

Harvey and Suraya Mattu (2017)

31 Smell of Data by

Leanne Wijnsma Studio (2016)

32 Tor Project by Roger Dingledine (2006)

33 The Alternet by Sarah T. Gold (2015)

34 Winston by Richard

Stokes (2019)

35 Counterbug by Erlend

Prendergast - Glasgow

School of Art (2019)
36 Accessories for the Paranoid by Pia-Marie Stute (2017)

37 Project Alias by Bjorn Karmann (2018)

38 CAPTCHA Tweet by Shinseungback Kimyong hun (2013) 39 Project Seen by Emil Kozole - Central Saint Martin (2015)

40 Open Informant by SuperFlux (2013); 41 Unfit Bits y Suraya Mattu (2015); 42 Google Maps Hacks by Simon Weckert (2020) 43 DNA Spoofiing $y$ Heather Dewey-Hangborg, Aurelia Moser, Allison Burtch and Adam Harvey (2013) 44 4G / WiFi / GPS Jammer (author unknown) 45 Nope Sound-Blocker by Ananda Svarupa Das (2017)

46 Webcam Cover (author unknown)

47 GPS Joystick Guide by The App Ninjas (2016) 48 QS1 Case by The Vysk 
Art-driven approach. Contemporary art often explores controversial issues within society, including the topic of surveillance. Artists such as Trevor Paglen, Ai Weiwei, Paolo Cirio, James Bridle, Laura Poitras have been exploring the theme of surveillance for years, creating works, exhibitions, performances, and objects related to this field. The artistic approach is generally characterized by a more satirical or dystopian tone that aims to make individuals aware of the theme of surveillance, for example, by dealing with issues concerning a world without privacy (Bringhenti, 2010). These works can have a "simple" purpose of denunciation or awareness-raising to artistic experimentation often associated with performances or provocative acts representing a form of subversion to surveillance.

Techno-scientific approach. Often, technological experimentation has greater degrees of freedom than R\&D activities aimed at the industrial production of objects or services that respond to the logic of the market economy. The research carried out can thus have different speculative purposes working on the experimentation of techniques that disrupt or prevent devices and systems from surveillance itself. One of the most relevant projects developed by the Belgian University KU Leuven designs specific textures to prevent facial recognition using digital neural networks.

Market-oriented approach. The existence of products dedicated to limiting surveillance shows that this issue is becoming mainstream due to scandals such as Cambridge Analytica and the Snowden case (Gellman et al., 2013; Cadwalladr \& Graham-Harrison, 2018). As a result, many users today find themselves in a critical or ambiguous situation: on the one hand, they understand the need to protect themselves; on the other hand, they increasingly find themselves having to use objects and devices for surveillance. In this regard, the spread of low-cost webcam cover shutters that allow users to obscure the camera of their laptop, smartphone or tablet is emblematic.

Design-driven approach. Compared to the art world, these projects have the appearance of objects or services in commerce. And it is precisely their possible existence on the market that facilitates users' understanding of the problem: seeing these objects as commercial objects pushes the user to ask questions about the current dynamics of surveillance, making him more aware of the issue (Dunne \& Raby, 2013). Within this field, one of the protagonists is Adam Harvey, author of CV Dazzle, Stealth Wear, and SkyLift.

Particularly, Critical Design can intervene on the theme of surveillance by subverting it through the creation of objects with which individuals and communities can protect themselves, or through objects representing the dystopia of a monitored world, hypothesizing objects and services possible only with the intent of ubiquitous surveillance (Malpass, 2017).

\section{Classific tion of Surveillance Subversion Methods}

From the extrapolation and subsequent analysis of only those cases working on subversion, it is possible to understand the main methods used. For this second level of study, has been created a second matrix to identify and describe four methods to surveillance 
subversion: Poisoning, Alerting, Deceiving, and Isolating Fig. 3. According to their reference technology and their application context, these methods can be declined in different ways: small or large scale, single object, or system.

Poisoning. The objects or services that use this method of subversion work by overloading the information we provide to specific devices or systems. Instead of hiding or covering up the data we generate, these products create an information flow characterized by an overabundance of data to make less accurate and reliable predictions about the individuals. The same thing happens in the Covid-19 tracking apps: Google and Apple have used Bluetooth technology to generate random IPs, intending to safeguard privacy automatically. The Poisoning method can be interpreted basically in two ways: overloading the device or lying to it. From the cases analysed, some objects that create an overload of information can provide incorrect information, thus "lying" to the technology. Another critical project in this field is Accessories for the Paranoid Fig. 5, which has challenged several surveillance devices using the Poison technique, such as laptop webcams, voice assistants, and digital devices in general.

Alerting. Fictional products such as Surveillance Spaulder and Anti-Nis Accessories aim to create awareness and design around the theme, devising objects that subvert by alerting the user to the presence of CCTV for the former and neural recognition technologies for the latter. The things designed with this method subvert the asymmetry of surveillance and provide the user with information about the surveillance itself. This information ranges from localizing surveillance objects to giving feedback on what our devices collect and how they classify or identify us. These objects allow us to understand when and how individuals are under surveillance, making the control tower of the Panopticon transparent, one might say.

Deceiving. This subversion method is strongly related to the concept of deceiving and cheating technologies, especially recognition technologies. In this area, one of the most relevant projects is Dazzle MakeUp by Adam Harvey Fig. 4. It is a platform that shares make-up techniques and hairstyles that protect the user by prevent- 
ing facial recognition. About facial recognition, the Face Dazzler app applies "adversarial" textures to the faces of photographs taken with the smartphone to impede facial recognition by third parties. These projects aim to limit automated machine recognition using Al-derived textures to fight $\mathrm{Al}$ and exploit bugs and gaps in recognition technologies.

Isolating. Another method for limiting surveillance is to intervene in the senses of surveillance itself by directly covering, hiding, and isolating surveillance objects. Concretely, the technique consists of covering, for example, the cameras through shutters or disabling microphones, etc. Despite this simplicity, the solutions can touch different fields and technologies, going from physical covers, such as shutters or white sounds to disrupt microphones, to fabrics and materials that shield Wi-Fi waves, GPS, 4G, etc. Examples in this area are Faraday Backpack, Off Pocket, and Embracing Paranoia, all three cases/backpacks for isolating one's connected electronic devices to limit surveillance, particularly geolocation.

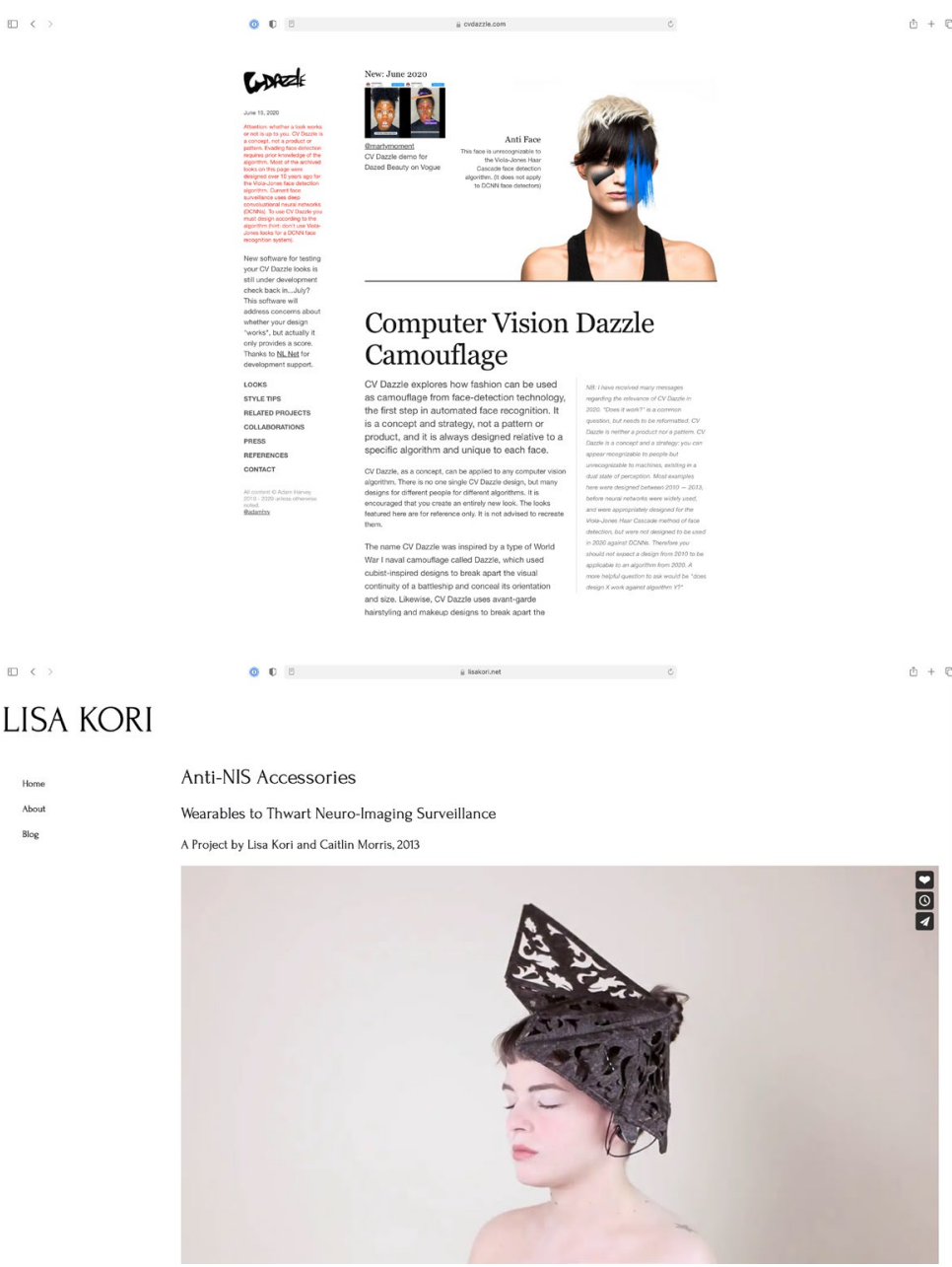

Fig. 4

Adam Harvey, CV Dazzle, 2010. Retrieved from: https://ahprojects.com/ cvdazzle/
Fig. 5

Lisa Kori and Caitlin Morris, Anti-NIS Accessories Wearables to Thwart Neuro-Imaging Surveillance, 2013. Retrieved from: https://lisakori.net/ anti-nis-accessories.html 


\section{Designing for Surveillance Resilience}

After analysing case studies from different fields and identifying methods and approaches used within creative processes aimed at subverting surveillance, a definition of Surveillance Resilience Design (SRD) has been proposed. It is a possible field of innovation that identifies and describes the principles and values on which to base the design of products and services for self-protection within the context of Ubiquitous Surveillance. SRD combines and integrates critical design techniques and methods with product design approaches and methods used in the arts, science, and technology. $\mathrm{SRD}$ is more closely related to Critical Design considered necessary to pose moral and ethical questions around the issue of ubiquitous digital surveillance, as opposed to the logic and practices of affirmative design. The purpose of identifying SRD principles and methods is to design objects to be adopted for user self-protection since the context of surveillance is not a speculative hypothesis but an existing situation. The projects created using SRD methods concern objects that are, at the same time, critical and usable by people to address their identified problems. In summary, Surveillance Resilience Design is an integrated approach to design that guides the creation of products and services that aim to protect individuals and communities from the Ubiquitous Surveillance and its dynamics of social classification and reduction of freedom of choice.

In conclusion, the first version of a Manifesto for Surveillance Resilience Design is proposed. It includes the general set of principles to guide the development of techniques and processes related to SRD.

SRD Principles:

- Protect from surveillance. Within the Surveillance Society, individuals should have the right to protect their privacy and, therefore, their freedom from the invasiveness of tracking and recognition technologies. This issue is not only a matter of principle but involves practical matters related to individual liberty. In this context, every piece of information can be used to classify and judge an individual, actively influencing their real life.

Subverting surveillance. The term "subvert" refers to the action of legally, independently, and autonomously intervening in an object or system with which degeneration of values is associated, actively or passively protecting oneself from surveillance practices. SRD aims to subvert surveillance practices, things, and technologies by exploiting the weaknesses and errors of the technologies themselves. Self-protection in the absence of institutional protection. Given the difficulty of implementing laws and activating fundamental protections by institutions, the only solution to protect oneself is to legally self-protect from surveillance. Therefore, a bottom-up resilience action can create self-producible or independently produced products-services to create awareness around the issue by stimulating the need for and importance of defining regulations or updating institutions. 
- $\quad$ Privacy but not digital hermitage. Designing services and products that allow individuals to protect their privacy without renouncing digital sociality, avoiding exclusion. Without access to devices like smartphones and their services, people are effectively excluded from significant parts of social life. In this sense, subversion can enable these devices and their services without creating exclusions from social context while maintaining control of our data.

- $\quad$ Resilience as a form of protection. The design aims to create functioning objects and services that make individual privacy protections for individuals or communities, subverting surveillance technologies to defend their rights.

- Resilience as a form of critique. The purpose of designing in this area is also to raise questions regarding the consequences of the Surveillance Society in a typical Critical Design approach.

- Legal Subversion. Surveillance Resilience Design should never exceed the limits of national and international laws. So as not to incriminate possible users and not encourage immoral behaviours.

SRD techniques and processes:

- "Poisoning" is confusing. Design products and services overload the data stream by confusing tracking and recognition technologies, making profiling more complicated and unreliable.

- Warn to understand. To design products and services enabling users to know surveillance and its objects better, rebalance the knowledge asymmetry between the monitored person and the surveillant to a minimum.

- Isolate to hide. Design objects and services that enable physical or virtual isolation of surveillance objects and technologies.

- Deceive to circumvent. Design objects and services that can deceive surveillance technologies to be circumvented so that they are not recognized or tracked by them.

\section{Conclusions}

The initial research hypothesis aims to investigate what and how design can stimulate solutions that support individuals to increase their awareness and agency concerning Ubiquitous Surveillance. The analysis of the literature has highlighted two main issues. Firstly, Ubiquitous Surveillance is an area of innovation in which a need for design emerges, but which cannot and should not be sole of a critical and speculative nature. Secondly, design is a discipline that has not yet developed a theoretical reflection accompanied by a practical application to operate in this context and generate an economical, productive, or social impact. The subsequent mapping of case studies reveals a plurality of approaches and modes of intervention favouring surveillance resilience. Still, it also highlights a difficulty in transforming these solutions into 
productive and usable product services, hinting at a possible "surveillance resilience market". Ironically, many of these solutions would be more likely to move out of exhibitions and art galleries and become the core content of functioning objects that can be easily made using open design and distributed production. Then, the Surveillance Resilience Design Manifesto is the first attempt to systematize experiences, design principles, and guidelines for all actors (internal and external to the design community) interested in designing product-service solutions for Surveillance Resilience. Overall, the entire work developed proposes an "in-progress framework" that attempts to link the theoretical part connected to the study of ubiquitous surveillance with the more experimental and project-based component represented by the cases. At the same time, the Manifesto can be seen as a "living document" whose principles and techniques can be revised, amended, and integrated. In design practice, the validity of SRD principles and techniques can be tested and challenged in the educational, scientific, or professional sphere or confronted with the regulations and rules of Privacy by Design. Finally, these principles and techniques could evolve into more sophisticated and interactive tools. It can be directly usable by citizens and organizations to interact more consciously with ubiquitous information systems, counter ubiquitous surveillance, and regulate or counterbalance asymmetries between human and non-human design agencies.
Massimo Bianchini Designer, Ph.D. in Design. Assistant Professor at the Department of Design and Lab Manager at Polifactory, the makerspace-FabLab of Politecnico di Milano. His research interests include open design and distributed production, small urban manufacturing, user-driven innovation, indie innovation, and circular innovation.

\section{Rei Morozumi}

Designer, MA in Integrated Product Design, Politecnico di Milano. He collaborated with Cilab Politecnico di Milano and the School of Design of Politecnico di Milano as a teaching assistant and coordinator of an international workshop on product development. His design activity focuses on products with a critical design approach. 
Bauman, Z., \& Lyon, D. (2013). Liquid Surveillance. A Conversation. Polity Press.

Beck, U. (2016). The Metamorphosis of the World. Polity Press.

Bringhenti, A.M. (2010). Artveillance: at the crossroads of art and surveillance. Surveillance \& Society, 7, 137-148.

Cadwalladr, C., \& Graham-Harrison, E. (2018). Revealed: 50 million Facebook profiles harvested for Cambridge Analytica in major data breach. The Guardian. https://www.theguardian. com/news/2018/mar/17/ cambridge-analytica-facebook-influence-us-election

DeCew, J.W. (1997). In Pursuit of Privacy: Law, Ethics, and Rise of Technology. Cornell University Press.

Dunne, A., \& Raby, F. (2013). Speculative Everything. Design, Fiction, and Social Dreaming. MIT Press.

Ferwerda, B., Schedl, M., \& Tkalcic, M. (2016). Using Instagram Picture Features to Predict Users' Personality. International Conference on Multimedia Modeling (pp. 850-861).
Fuad-Luke, A. (2009).

Design Activism. Beautiful

Strangeness for a Sustainable World. Routledge.

Galič, M., Timan, T. \& Koops, B.J. (2017). Deleuze and Beyond: An Overview of Surveillance Theories from the Panopticon to Participation. Philos. Technol, 30.

Gandy, O.H. (2016). Coming to Terms with Chance Engaging Rational Discrimination and Cumulative Disadvantage. Routledge.

Gellman, B., Blake, A., \& Miller, G. (2013). Edward Snowden comes forward as source of NSA leaks. The Washington Post. https:// www.washingtonpost.com/ politics/intelligence-leaders-push-back-on-leakers-media/2013/06/09/ fff80160-d122-11e2-a73e826d299ff459_story.html

Harari, Y.N. (2015). Homo Deus. A brief history of tomorrow. Harvill Secker.

Henkin, L. (1974). Privacy and Autonomy. Columbia Law Review, 74(8), 14101433.

Kelly, K. (2016). The Inevitable. Understanding. The 12 Technological Forces that will Shape our Future. Viking Press Penguin Books.
Khatchadourian, R. (2015).

We Know How You Feel.

Computers are learning

to read emotion, and the

business world can't wait.

The Newyorker. https://

www.newyorker.com/magazine/2015/01/19/know-feel

Kumar, P.C. (2019). A

Post-Humanist Take on

Surveillance Capitalism.

Proceedings of $\mathrm{CHI}$ Conference on Human Factors (CHI 2019). ACM.

Latour, B. (2005). Reassembling the Social: An Introduction to Actor-Network-Theory. Oxford University Press.

Malpass, M. (2017). Critical Design in Context. History, Theory and Practices. Bloomsbury.

Mann, S., Fung, J., Federman, M., \& Baccanico, G. (2003). PanopDecon: deconstructing, decontaminating, and decontextualizing panopticism in the post-cyborg era. Surveillance \& Society, 1(3).

Pötzsch, H. (2014). FCJ-172 Posthumanism, Technogenesis, and Digital Technologies: A Conversation with N. Katherine Hayles. The Fibreculture Journal, 23.
Oulasvirta, A., Pihlajamaa, A., Perkiö, J., Ray, D., Vähäkangas, T., Hasu, T., Vainio, N., \& Myllymäki, P. (2012). Long-term effects of ubiquitous surveillance in the home. UbiComp '12: Proceedings of the 2012 ACM Conference on Ubiquitous Computing ( $\mathrm{pp}$. 41-50).

Schwartz, H.A. (2016). Predicting Individual Well-being through the Language of Social Media. Pacific Symposium on Biocomputing (pp. 516-527).

Wong, R.Y., \& Mulligan D.K., (2019). Bringing Design to the Privacy Table: Broadening "Design" in "Privacy by Design" Through the Lens of $\mathrm{HCl}$. CHI '19: Proceedings of the 2019 CHI Conference on Human Factors in Computing Systems (pp. 1-17).

Zuboff, S. (2018). The Age of Surveillance Capitalism: The Fight for a Human Future at the New Frontier of Power. Public Affairs. 